\title{
Experimental and FEM based investigation of the influence of the deposition temperature on the mechanical properties of SiC coatings
}

\author{
Thomas SCHLECH ${ }^{a,}$, Siegfried HORN ${ }^{b}$, \\ Charles WIJAYAWARDHANA ${ }^{c}$, Arash RASHIDI $^{a}$ \\ ${ }^{a}$ SGL Carbon GmbH, Werner-von-Siemens-Strasse 18, 86405 Meitingen, Germany \\ ${ }^{b}$ Experimental Physics II, Institute of Physics, University of Augsburg, 86135 Augsburg, Germany \\ ${ }^{c} S G L$ Carbon GmbH, Drachenburgstrasse 1, 53170 Bonn, Germany
}

Received: February 26, 2020; Revised: October 12, 2020; Accepted: October 15, 2020

(C) The Author(s) 2020.

\begin{abstract}
Scanning electron microscopy shows that the microstructure, in particular the overall grain size, of chemical vapor deposited silicon carbide coatings depends on the deposition temperature. So far, the influence of the microstructure on the mechanical properties of such coatings is not well described in literature. To investigate the influence of the deposition temperature on the mechanical properties of the coating, nanoindentation is used in this work. Since the measurement results of nanoindentation can be affected by the substrate material, the contribution of the substrate material is taken into account utilizing a finite element model. The model is then employed to generate information about elastic and plastic properties of the coating by inverse simulation. To evaluate the fracture toughness of the coating, the generated material model is used in a cohesive-zone based formulation of the fracture process during indentation at higher loads. The results of this model allow determining the fracture toughness of silicon carbide coatings deposited at different temperatures.

Keywords: nanoindentation; finite element analysis; coatings; ceramics; fracture mechanics
\end{abstract}

\section{Introduction}

A broad range of mechanical properties is reported for silicon carbide $(\mathrm{SiC})$ in the literature. For chemical vapor deposited (CVD) $\mathrm{SiC}$ coatings the Young's modulus values range from around $330 \mathrm{GPa}$ [1] up to approximately $480 \mathrm{GPa}$ [2]. Reasons for this broad range in the reported data can result from different measurement methods, experimental errors, or from different production processes of the coatings and, therefore, differences in their microstructure. The

* Corresponding author.

E-mail: thomas.schlech@gmx.de, thomas.schlech@sglcarbon.com influence of coating process parameters on the microstructure has been already described [3,4]. Chin et al. [5] have shown systematically that the morphology, crystal structure, and chemical composition of CVD $\mathrm{SiC}$ depend on the deposition temperature, the pressure in the reactor, and the ratio of precursor to carrier gas. The work of Gulden [6] suggests that, the deposition temperature primarily influences the microstructure of the SiC-coating, while the pressure and gas ratio have only a minor effect. While the dependence of the microstructure on the deposition temperature is well documented, the influence of microstructure on the mechanical properties is rarely investigated. Since the existing literature suggests that, the deposition 
temperature is the most dominant parameter for the microstructure, in this work we investigate the influence of deposition temperature on the mechanical properties of $\mathrm{SiC}$ coatings.

Instrumented indentation, also called nanoindentation, is a powerful tool to characterize coatings and thin films, since the sample volume can be kept quite small. However, if the standardized Oliver and Phar method [7] for bulk materials is used, the measurement of the elastic modulus can be affected by the respective substrate. To extract reliable values, a FEM model is set up in this work to account for the substrate effect applying an inverse analysis procedure. The necessity to account for the contribution of the substrate material is justified by the fact that the combination of $\mathrm{SiC}$ coating and graphite substrate is a hard and stiff coating on a soft substrate. In addition to elastic properties, which are also evaluated by the Oliver and Phar method, this allows us to generate information about the plastic deformation of the coating. The generated elastic-plastic material model is used in further simulations to evaluate the fracture toughness of the coatings.

\section{Materials and methods}

\section{1 Samples and experimental setup}

In the work presented five different samples are investigated. Graphite substrates were coated via chemical vapor deposition, in an industrial CVD reactor at three different deposition temperatures. In addition to the deposition temperature, the coating thickness of the coating deposited at the medium temperature was varied. The different coating thicknesses were achieved by varying the deposition time. A clear diverse appearance of the surface of $\mathrm{SiC}$ coatings deposited at different temperatures was observed by scanning electron microscopy (SEM). While the coating deposited at lower temperatures showed a smoother and cauliflower like appearance, the coating surface produced at higher temperatures consisted of clearly visible crystallites, as shown in Figs. 1(a)-1(c). For the SEM investigation we used the secondary electron detector due to a higher surface sensitivity.

Consistent with the diverse appearance of the coating surface, the cross-sectional images also revealed significant differences in the coating microstructure. The coating deposited at lower temperatures had a

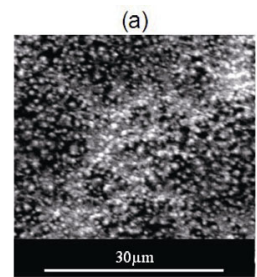

(d)

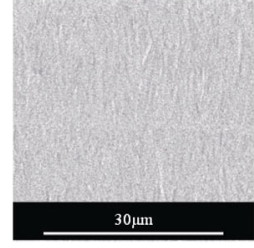

Fig. 1 Surface scanning electron microscopy of silicon carbide coating with different deposition temperatures: (a) lowest, (b) medium, and (c) high; cross-sectional scanning electron microscopy images at different temperatures: (d) lowest, (e) medium, and (f) high.

much finer grained structure than the coatings deposited at higher temperatures, depicted in Figs. 1(d)-1(f). For these images, the backscattered electron detector (BSD) was used, since it led to a better contrast between the differently oriented grains. The different gray value of the BSD images is caused by different grain orientations, besides the pores in the material. This was also published by other authors who additionally used electron backscatter diffraction (EBSD) [8]. In their work, the different gray value from SEM and grain orientations from EBSD correlated very well. Via X-ray diffraction and energy-dispersive X-ray spectroscopy no inclusions, different phases, or reasonable impurities were observed in the coating.

For nanoindentation all samples were embedded in an epoxy matrix, polished, and subsequently finished with a $1 \mu \mathrm{m}$ diamond suspension, to ensure a smooth surface. A smooth surface reduces the influence of surface roughness on the nanoindentation measurement [9]. For all experiments the nanoindenter UNAT from former ASMEC GmbH (now Zwick/Roell) was used. All measurements were performed quasi-static according to DIN EN ISO 14577-1 [9], force controlled, and with a maximum load of $25 \mathrm{mN}$ using a Berkovich type indenter tip. Higher forces were not applied to avoid cracking of the $\mathrm{SiC}$ coating. Measurements were performed in cross-section and normal to the coating surface at each sample as depicted in Fig. 2.

At least 50 different locations were measured for each sample, to get a statistically significant average value. The measured data were evaluated using DIN EN ISO 14577 [9] as well as the inverse method using a finite element model. For the inverse analysis, a mean value curve of all 50 measurement locations is used as input. 


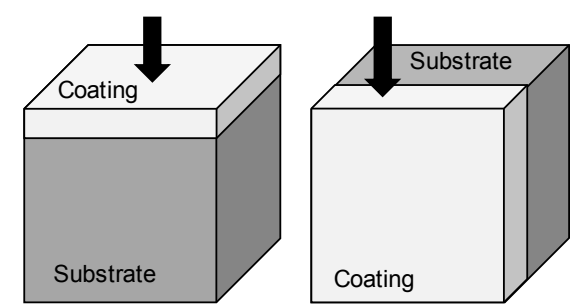

Fig. 2 Schematic of the cross-sectional measurement (a) and the measurement normal to the coating surface (b).

For the fracture toughness measurement, it was not possible to use the indents from the elastic modulus measurement, since pronounced cracking of the sample is required for the fracture toughness test. The pronounced cracking was achieved in further experiments using a maximum load of $1 \mathrm{~N}$. The crack lengths were evaluated using scanning electron microscopy. For the measurement of the crack lengths 10 measurements per were performed for each sample, producing 3 cracks per imprint in each corner of the indent. The fracture toughness was calculated using the results of a cohesive-zone based FEM model of the indentation fracture.

\section{2 Measurement of elastic modulus}

\subsubsection{Measurement principle}

The hardness and elastic properties of materials can be measured by nanoindentation on a nanometer and micrometer scale. This makes nanoindentation an attractive measurement method for the characterization of thin films. The measurement method itself is based on the measurement of the load displacement curve during loading and unloading of a sample using an indentation tip of known geometry. A typical force displacement curve is illustrated in Fig. 3.

To test materials in the elastic-plastic region we used a Berkovich type indenter tip. The force-displacement response in the measured region is determined

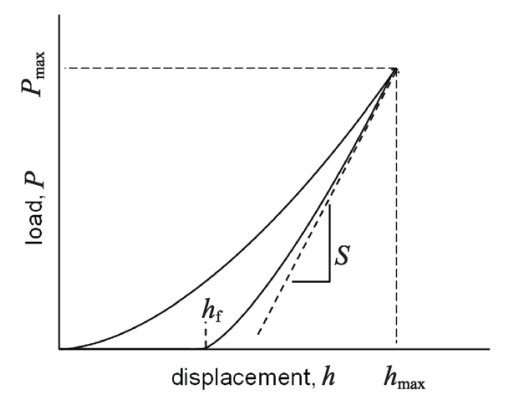

Fig. 3 Force displacement curve of a typical nanoindentation experiment. by the elastic-plastic behavior of the investigated material during loading. During unloading, only the elastic recovery of the sample determines the force displacement curve [10], until the indenter tip loses contact with the sample at the remanent displacement $h_{\mathrm{f}}$. To determine Young's modulus $E$ by the standardized method of Oliver \& Pharr [9] the contact stiffness is taken as the slope of the unloading curve at the maximum applied load (see Section 2.2.2).

\subsubsection{Analytical model by Oliver \& Pharr}

The analytical model by Oliver \& Pharr $[10,11]$ is the most common method to determine the hardness and elastic modulus by instrumented indentation and is accepted as a standardized method [9]. The used quantities for evaluation of the Young's modulus are the maximum load $P_{\max }$, the maximum indentation depth $h_{\max }$, the remanent displacement $h_{\mathrm{f}}$, and the elastic unloading stiffness $S$. The unloading stiffness is determined by the derivative of a fit function of the unloading curve at $h_{\max }$ as shown in Fig. 3 . The fit function $P(h)$, is chosen [10] as a power law of the form:

$$
P(h)=\alpha\left(h-h_{\mathrm{f}}\right)^{m}
$$

where $\alpha$ and $m$ are best fit values. Consequently, the unloading stiffness can be expressed as

$$
S=\left.\frac{\mathrm{d} P}{\mathrm{~d} h}\right|_{h_{\max }}
$$

The periphery of the indent deforms during the indentation as well as visualized in Fig. 4.

This so-called "sink in" of the periphery can be assumed to be purely elastic and thus can be described by models of the indentation of a flat elastic half space by a rigid punch. Such a model is treated in the work of Sneddon [12]. Taking the deformation of the periphery $h_{\mathrm{s}}$ into account, the contact depth $h_{\mathrm{c}}$ can then be calculated by subtracting the sink in of the periphery from the maximum indentation depth such that

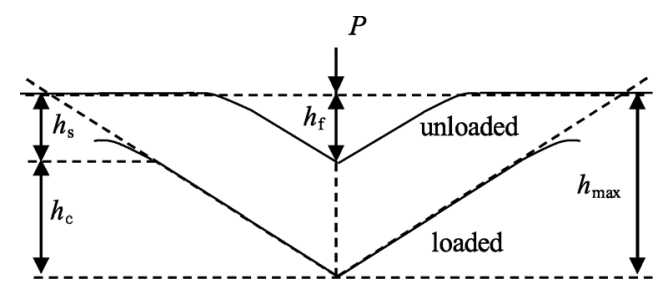

Fig. 4 Schematic illustration of parameters describing the contact of the indenter with the sample at loaded and unloaded state according to Ref. [10]. 


$$
h_{\mathrm{c}}=h_{\max }-h_{\mathrm{s}}=h_{\max }-\epsilon \frac{P_{\max }}{S}
$$

The constant $\epsilon$ depends on the indenter geometry. For a Berkovich type indenter, a value of $\epsilon=0.75$ can be used as a standard value [13]. It can be adopted, as described in DIN EN ISO 14577 [9], to improve the accuracy of the indentation depth measurement and indenter area function.

Pharr and Oliver have shown that for different contact geometries, a simple relationship between contact stiffness, contact area, and the effective elastic modulus, often called reduced modulus, exists. For all contacts, which are governed by Sneddon's contact theory [10], following expression for the effective modulus $E_{\text {eff }}$ is applicable [13]:

$$
E_{\text {eff }}=\frac{S}{2 a}
$$

where $a$ is the contact radius of an axis-symmetric indenter with the same projected contact area as the Berkovich indenter. For a precise measurement of the hardness and Young's modulus it is important to be able to determine the contact area between indenter and sample during the measurement. The contact area $A$ is then used to calculate an effective value for the contact radius $a$ by

$$
a=\sqrt{\frac{A}{\pi}}
$$

Since the contact area $A$ between the indenter and the sample cannot be measured directly, it is being calculated by its indenter shape function. The indenter shape function is a function of $h_{\mathrm{c}}$ and must be calibrated in independent measurements. A method to determine such a shape function can also be found in the work of Oliver and Pharr [10].

Based on this method the effective elastic modulus can be calculated by substituting Eq. (5) in Eq. (4) yielding

$$
E_{\mathrm{eff}}=\frac{S \sqrt{\pi}}{2 \sqrt{A}}
$$

The effective elastic modulus calculated with the help of Eq. (6) does not correspond to the sample's elastic modulus. Using the known modulus $E_{i \mathrm{~d}}$ and the Poisson's ratio $v_{i}$ of the indenter and the Poisson's ratio of the sample $v$, the system can be treated analogous to springs in a serial arrangement. For the calculation the plain strain modulus $\frac{E}{(1-v)^{2}}$ of the sample and
$\frac{E_{i}}{\left(1-v_{i}\right)^{2}}$ of the indenter are used [7,14].

The elastic modulus of the sample can then be calculated using [7]:

$$
\begin{gathered}
\frac{1}{E_{\mathrm{eff}}}=\frac{1-v^{2}}{E}+\frac{1-v_{i}^{2}}{E_{i}} \\
E=\left(1-v^{2}\right)\left(\frac{1}{E_{\mathrm{eff}}}-\frac{1-v_{i}^{2}}{E_{i}}\right)^{-1}
\end{gathered}
$$

\subsubsection{FEM model-modulus determination}

The original Oliver \& Pharr method has been initially developed for bulk materials. Thus, the applicability of the method is questionable for very thin coatings or coatings deposited on substrates showing significant different mechanical properties. This is due to the fact that, no substrate material is considered in the evaluation of the original Oliver \& Pharr method. Since SiC is a highly stiff material compared to the graphite substrate, the influence of the substrate can lead to apparent low moduli using the method of Oliver and Pharr. Evaluation of the elastic properties via inverse FEM analysis can overcome this problem. This is achieved by including the substrate in the FEM model. Using ANSYS workbench [15], the model presented here is created under the assumption that a continuum mechanics description is appropriate for indentation depths beyond 10-20 $\mathrm{nm}$ [16]. The coating is modeled as a continuum, i.e., its microstructure is not taken into account.

To reduce computational effort and time, the problem is modeled as an axis symmetric 2D problem, representing the tip as a conical shape $[17,18]$ as illustrated in Fig. 5.

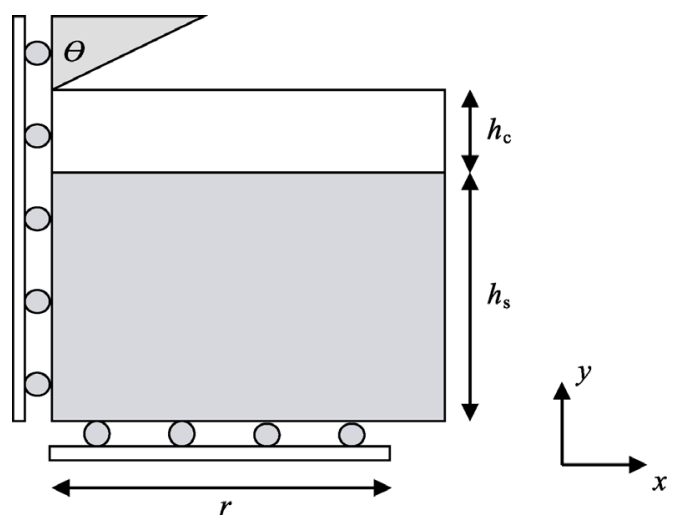

Fig. 5 Schematic of the 2D axisymmetric FEM model. 
To simulate the instrumented indentation experiments the model geometry must be sufficiently large to avoid the influence of the boundary effects, but as small as possible to reduce the computational effort. The real tip geometry can differ from the ideal shape due to small deviations during the fabrication of the indenter tip or tip rounding caused by wear of the indenter. The indenter geometry was generated using the area function of the indenter. This means that the radius of the cone was expressed as a function of contact depth using Eq. (5). The indenter material was modeled as linear elastic material with material properties commonly used for diamond ( $E=1140 \mathrm{GPa}, v=0.07)$ [19].

The symmetry axis and the horizontal boundary were modeled as frictionless supports. The indentation of the tip can be realized by a forced displacement of the top horizontal edge of the indenter or an applied force. Since our experiments are force-driven, the model of the indentation process is realized by a boundary force. As boundary condition a remote force boundary condition was chosen. This means, that a force was applied to a remote point whose degree of freedom solution is coupled with the elements of the upper vertical edge of the indenter. This ensured a rigid behavior of the top horizontal edge in loading direction.

The contact between sample and tip is modeled with contact elements accounting for frictionless contact. The contact stiffness was chosen sufficiently high to avoid contact penetration.

To obtain the necessary information to calculate the force displacement curve, the contact force was evaluated as the reaction force of the bottom elements in vertical direction. To get depth values comparable with the experimental data, one should keep in mind that, the experimentally determined force displacement curve includes both the deformation of the indenter and the sample, as the frame stiffness does not account for the deformation of the diamond tip. This might be negligible for the indentation of materials with a much lower stiffness than diamond. Since we address silicon carbide the deformation of the diamond tip cannot be neglected. The indenter deformation was taken into account using the absolute value of the directional displacement of the indenter top horizontal edge to account for the lateral and vertical deformations of the indenter $[17,19]$.

To account for the plastic deformation of the sample, which is inevitably connected with the indentation process using a sharp indenter, a constitutive law including plastic deformations is needed. The sample was modeled as isotropic elastic perfectly plastic material, with no hardening effects included. This is a reasonable assumption for indentation modelling of amorphous or nonmetallic materials [16]. For this reason, the bilinear isotropic hardening material model already implemented in ANSYS [15] was used. As input for this constitutive model, the Young's modulus, Poisson's ratio, and the yield strength are needed. Since the material is assumed perfectly plastic, there is no hardening, and hence the tangent modulus is chosen to be zero.

The substrate was modeled as isotropic linear elastic material, characterized by dynamic modulus measurement. The Young's modulus used for the substrate was 10.5 GPa and the Poisson's ratio was chosen to be 0.14 .

\subsubsection{Inverse simulation procedure}

Several different approaches for the inverse material property determination via FEM can be found in literature $[16,20]$. They all have in common that the Young's modulus and the yield strength of the material can be fitted to the experimentally measured force displacement curve.

The material models used in the FEM model are sometimes not sufficient to describe both the unloading and loading process perfectly. This is due to an overly simplification of the plastic material behavior. Additionally, creep effects at maximum indentation force may impede a proper fit to the combined loading and unloading curve [16].

Yu et al. [20] suggested fitting the material using the experimental loading curve only. This is a reasonable approach if mainly the plastic material behavior is of interest or if the elastic properties of the material are already known. However, if the elastic response of the material is in the focus of the investigation a fit to the loading curve only can lead to poor results. In this case, fitting to the unloading curve only appears well suited. This corresponds approximately to the analysis of Oliver \& Pharr, where also only data of the unloading curve are used.

Knapp et al. [16] showed a more advanced method, where the maximum force and maximum deformation as well as the unloading stiffness are used for inverse analysis. This method has the advantage that creep effects at maximum load are decoupled from the model. 
The present study suggests that a fit to the unloading data is sufficient to describe elastic properties of $\mathrm{SiC}$ coatings on graphite substrates. This is due to the fact that creep effects are not expected, and therefore, the implementation of a more complex model like the model of Knapp et al. is not needed.

The fitting itself is performed using a least square fit of 10 calculated values to the corresponding experimental data. The optimization problem is solved using the direct optimization tool in ANSYS DesignXplorer [15].

\subsection{Measurement of fracture toughness}

\subsubsection{Measurement principle}

The origin of the method is described in the work of Palmqvist, who investigated cracks caused by Vickers indentation. In his work he related the length of the crack to the toughness of the material [21]. During indentation mainly two crack types namely the halfpenny crack [22] and the radial or Palmqvist crack [21] can be experimentally observed for different materials. A schematic of both crack types is shown in Fig. 6. The formation of half penny cracks is generally expected for low toughness materials under high loads, while the Palmqvist cracks are expected for tougher materials under lower loads [23].

The work of Palmqvist [21] was followed by the work of Lawn et al. [22,24-26]. Experiments of Lawn and Marshall gave a better insight into the crack morphology developing in glass during loading and unloading using a Vickers indenter [27]. They came to the result that not only the tensile stress generated by the elastic contribution of the indentation during loading plays an important role in crack formation, but also the residual stress remaining during unloading. Further indentation experiments were directed to the quantification of the toughness. First results were successfully achieved by Lawn and Fuller [26], who showed, that the crack length increases to the power of $2 / 3$ with increasing load $P$. To determine the fracture

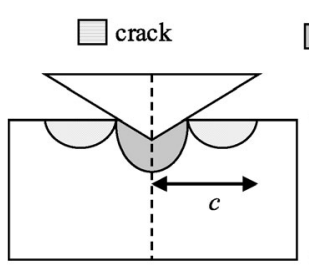

(a)

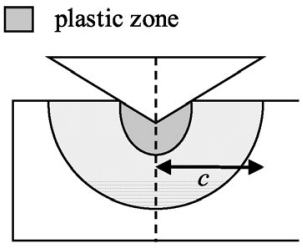

(b)
Fig. 6 Crack types: (a) Palmqvist/radial crack, (b) half penny crack. toughness by indentation techniques, it was found that both the hardness and the elastic modulus of the material play a major role besides crack length and load. From this basic observation empirical relations between the four involved parameters were deducted [28]. A description of a halfpenny shaped crack was developed by Lawn et al. [22]. Their model assumes that during loading, the elastic contact stress and the residual stress caused by plastic deformation cause crack growth, while during unloading only the residuals stress plays a role for crack growth. Superposition of both effects results in the observed crack growth. Using analytical approaches for the residual stress component and the elastic component they showed that the stress intensity factor scales with the residual stress component in the same way as the load and crack length. Both components were incorporated in one equation, with an empirical fitting constant that was determined by Anstis et al. [24]

$$
K_{\mathrm{Ic}}=\alpha\left(\frac{E}{H}\right)^{\frac{1}{2}} \frac{P}{c^{\frac{3}{2}}}
$$

Here $\alpha$ is an empirical fitting constant, $E$ the Young's modulus, $H$ the hardness, $P$ the applied load, and $c$ the distance from the crack tip. In most cases a value of 0.016 is used for $\alpha$, resulting from the work of Anstis et al. for a Vickers indenter, calibrated for several different materials [24]. Jang and Pharr [29] investigated the validity of the equation later for indenter tips with different geometries on bulk $\mathrm{Si}$ and Ge. The result was a refined description of the fitting constant $a$ as a function of the half opening angle of the indenter $\Psi$ :

$$
\alpha=\frac{0.0352}{1-v}(\cot \Psi)^{\frac{2}{3}}
$$

There are also other semi-empirical models to evaluate the fracture toughness via indentation. An overview of the different approaches is given in a review of Quinn and Bradt [30]. Since in the model of Anstis et al. [24] $\alpha$ is only calibrated for the usage of a Vickers indenter, while Jang and Pharr calibrated their model only for $\mathrm{Ge}$ and $\mathrm{Si}$, in this work a finite element model of the indentation fracture process is used for the calibration of $\alpha$.

For $\mathrm{SiC}$ coatings the generation of residual stress due to high temperature deposition processes results in an additional problem. The residual stress, if large 
enough, may have a non-negligible influence on the fracture toughness measurement. To account for the residual stress, Eq. (8) can be modified to [25,31]:

$$
K_{\mathrm{Ic}}=\alpha\left(\frac{E}{H}\right)^{\frac{1}{2}} \frac{P}{c^{\frac{3}{2}}}+Z \sigma_{\mathrm{r}} \sqrt{c}
$$

where $Z$ is a function of the crack shape and has the value 1.26 for an ideal halfpenny crack [31].

\subsubsection{FEM model-indentation fracture toughness}

A FEM model was applied to obtain a deeper understanding of the induced cracking during indentation fracture testing and to calibrate the semi-empirical fitting constants. In contrast to the above discussed model for elastic modulus determination, a 2D axisymmetric model is not sufficient in this case, since the stress concentration at the edges of the Berkovich indenter plays a non-negligible role in the formation of cracks. Thus, a 3D model is developed.

In this $3 \mathrm{D}$ model the indenter was described as a perfectly shaped rigid Berkovich tip. The sample was modeled as two times one sixth of a cylinder, coupled via cohesive zone elements as depicted in Fig. 7.

The indentation load was applied as remote force boundary condition. The contact between the indenter and the sample is chosen as frictionless as in the $2 \mathrm{D}$ model. The cohesive law is chosen as bilinear traction separation law illustrated in Fig. 8.

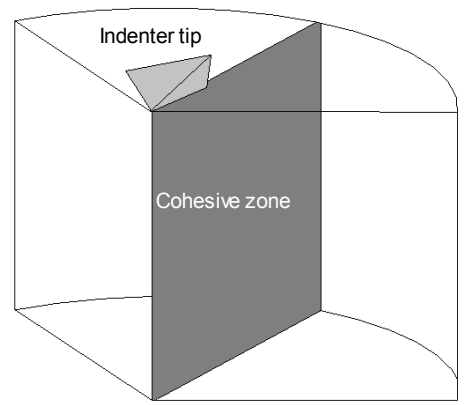

Fig. 7 Schematic of the geometry of the 3D FEM crack model.

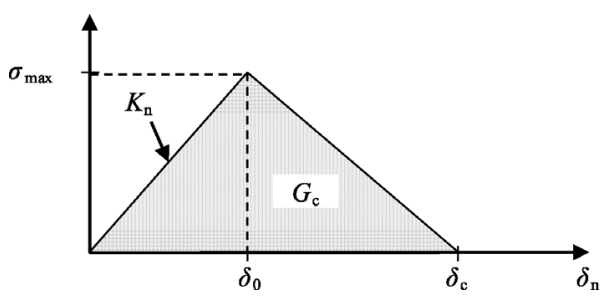

Fig. 8 Bilinear traction-separation law for the cohesive zone.
As input parameters for the traction separation law the critical strain energy release rate $G_{\mathrm{c}}$ and the cohesive strength $\sigma_{\max }$ were used, from which the critical decohesion length $\delta_{\mathrm{c}}$ can be calculated via

$$
\delta_{\mathrm{c}}=\frac{2 G_{\mathrm{Ic}}}{\sigma_{\max }}
$$

For brittle materials, the initial contact stiffness $K_{n}$ ideally should be infinitely high as proposed by different authors [32,33]. In a numerical simulation an infinite initial contact stiffness cannot be realized. Therefore, the initial contact stiffness must be chosen to be high enough to be physically meaningful and small enough to ensure convergence of the FEM model.

The cohesive strength $\sigma_{\max }$, can be chosen in different ways. The most straight forward approach would be to estimate $\sigma_{\max }$ from the known macroscopic tensile strength. But this may lead to poor results, since $\mathrm{SiC}$ is expected to have a much higher strength at the smaller scales in focus here based on Weibull's weakest link model [34,35].

$\mathrm{SiC}$ can be assumed to show brittle behavior and should therefore deform elastically until failure, without a huge amount of plastic deformation before complete decohesion. Therefore, the critical decohesion length has to be quite small. Using the bilinear traction separation law implemented in Ansys workbench [15] the only possible way to ensure a small critical decohesion length $\delta_{\mathrm{c}}$, with fixed $G_{\mathrm{Ic}}$ is increasing the cohesive strength value. One can choose a strength value related to the atomic bonding strength, to capture the crack propagation best. Using the atomic bonding strength nevertheless leads to numerical problems. One problem is that a very fine mesh would be required to describe the nearly singular stress state around the crack tip. The other problem is that sufficiently low load increments would be needed to describe the cohesive behavior. Both combined would lead to a very high computational effort.

As a solution we used a cohesive strength value higher than the macroscopic strength, or strength scaled down to the test volume by Weibull's weakest link model. This is a reasonable approach, since the exact crack onset is not main topic of the model used here. To circumvent the related problems with high cohesive strength values, the theoretical strength value is also not chosen. It was found that, after a sufficient small process zone is reached, and the related brittle 
behavior is achieved, further increase of the strength does not change the results in a significant way. Only the computational effort would increase. In our case it has been shown that approximately $3 \mathrm{GPa}$ for $\sigma_{\max }$ lead to reasonable results.

Since the aim of the investigation is to measure the fracture toughness of the coating the FEM model was used to calculate base points to generate a function which correlates the fracture toughness to the length of the cracks generated by indentation. The function can then be compared with the semi-empirical equations from literature and can be used to calibrate the semi empirical fitting constant.

\section{Results}

\section{1 Measurement of the indentation modulus}

\subsubsection{Cross-sectional measurement}

It is initially assumed that, for cross-sectional measurements the coating thickness has no influence on the measured modulus using the method of Oliver and Pharr. To check this assumption three different coatings produced at the same conditions with different thicknesses were tested in cross section. Figure 9 shows that the results do not differ significantly for different coating thicknesses, although the modulus of the thinnest coating seems to shift to somewhat lower values.

For thin coatings, measured in a cross-sectional setup, it can be shown that the elastic modulus determined by the Oliver \& Pharr method decreases, which is a result of neglecting the nature of the indentation periphery. To check the influence of the substrate on the cross-sectional measurement, a threedimensional FEM model was used. The coating thickness in the model was parametrically changed and the resulting force displacement curves from the FEM model were analyzed via the analytical Oliver \& Pharr

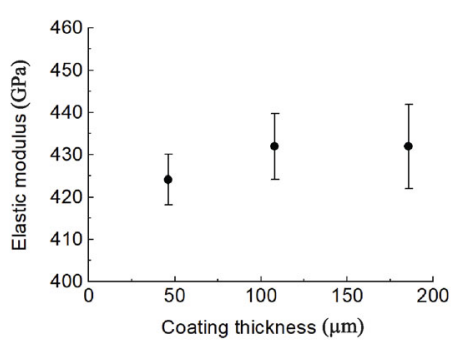

Fig. 9 Modulus measured in cross section at different coating thicknesses using Oliver \& Pharr method. method. For the SiC-coated graphite material system, the influence of the substrate thickness becomes reasonable for coatings thinner than $50 \mu \mathrm{m}$. For the thinnest coating from Fig. 9 this would cause a shift of about $2.5 \%$ shifting the Young's modulus of the thin coating to the same level as the thicker coatings.

Since the coating thickness in the cross-sectional measurements shows no significant influence on the measured modulus, the analytically determined values for the coatings deposited at different temperatures can be directly compared. The value of the modulus, evaluated by standardized analysis for bulk materials [9] are depicted in Fig. 10. It can be seen that the coatings deposited at lower temperatures show a significant higher modulus, while medium and the highest used deposition temperature show no significant difference between each other.

\subsubsection{Measurement normal to the coating surface}

To test the accuracy of the model for the inverse analysis, coatings deposited at the same temperature but with different thicknesses are investigated via instrumented indentation normal to the coating surface. The analysis of the experimental data is performed using the Oliver \& Pharr method as well as the inverse procedure. The experimentally determined force displacement curves as well as the best fit data from the inverse analysis are depicted in Fig. 11.

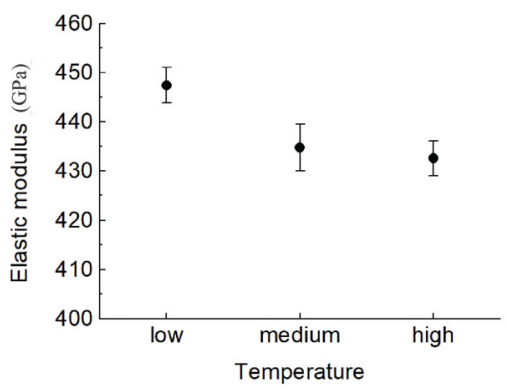

Fig. 10 Cross-sectional determined Young's modulus for coatings deposited at different temperatures.

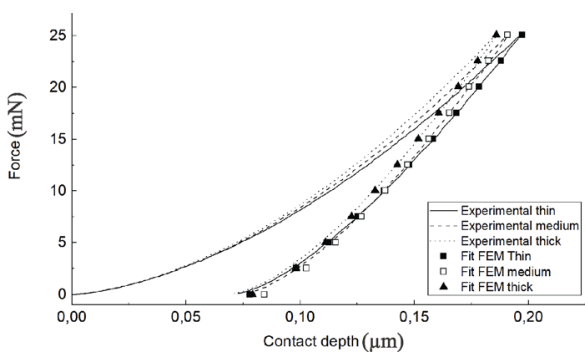

Fig. 11 Force displacement curves measured normal to the coating surface (dotted lines) and best fit result from the FEM model for the measurement of coatings with different thicknesses deposited at the medium temperature. 
Based on the cross-sectional measurements the Young's modulus is not expected to show significant difference in the range of the varied coating thickness. Nevertheless, the standardized evaluation for bulk material leads to visible differences in the results for the coatings with different thickness, shown in Fig. 12, which is most likely caused by the influence of the substrate.

It can be seen in Fig. 12 that the inverse analysis procedure is capable of fully taking into account the influence of the substrate on the measurement, since a uniform modulus over all coating thicknesses after including corrections by the inverse modeling approach is observed.

Since the inverse procedure leads to reliable results, it can also be used to evaluate the modulus of the coatings deposited at different temperatures.

The best fit results from the inverse model to the experimentally determined force displacement curves for the coatings deposited at different temperatures are shown in Fig. 13. A very good agreement between the computed data and the experimental data is achieved.

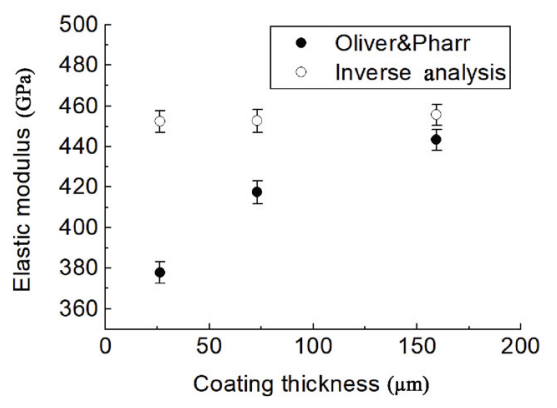

Fig. 12 Comparison of the modulus measured normal to the coating surface at different coating thicknesses using the analytic Oliver \& Pharr method and the inverse analysis procedure.

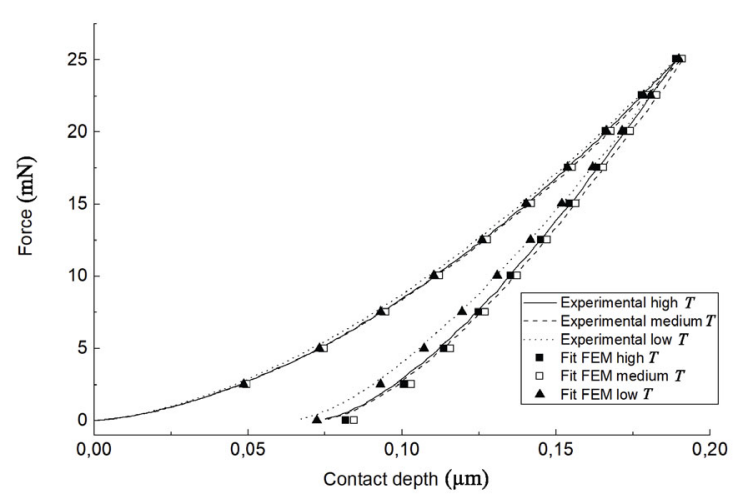

Fig. 13 Measured force displacement curves (dotted lines) and best fit result from the FEM model for the measurement of coatings with different thicknesses and deposition temperatures.
The major difference of the curves is the slope of the unloading curves at the maximum load, which are used for the evaluation. The change of slope at this point is not easily detectable by bare eye. The offset on the $y$-axis is mainly caused by hardness differences of the coating, not the modulus. The results of the inverse procedure and of the Oliver \& Pharr method for the coating modulus normal to the coating surface are shown in Fig. 14.

The obvious difference between the Oliver \& Pharr method and the here used inverse analysis is caused by different coating thicknesses of the coatings deposited at different temperatures. The coating deposited at low temperatures also showed the smallest coating thickness. The lower coating thickness leads to a higher influence of the substrate elastic behavior and consequently to lower apparent coating stiffness relying on the Oliver \& Pharr method for bulk materials. The inverse procedure in contrast to the standard Oliver \& Pharr method accounts for this influence.

Consistent with the results from the cross-sectional measurement, the modulus normal to the coating surface shows a higher value for the coating deposited at the lowest temperature while again the coatings deposited at medium and the highest temperature exhibited nearly the same modulus.

\section{2 Fracture toughness measurement}

The measurement of the fracture toughness is only performed normal to the coating surface. This is due to the higher complexity of cross-sectional measurements. The higher complexity arises from the non-equibiaxial stress state at the indented surface at cross-sectional measurements, which is due to residual stress distribution in the coating. For the fracture toughness measurements, the same samples were used as for the modulus measurement.

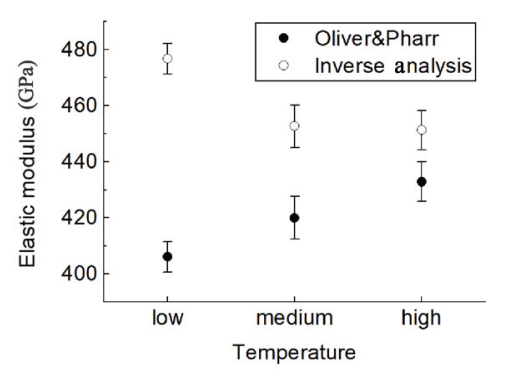

Fig. 14 Comparison of the modulus measured normal to the coating surface at coatings with different deposition temperature using Oliver \& Pharr method and the inverse analysis. 
Cracks were initiated by indentation and then characterized via scanning electron microscopy. To investigate a possible influence of the maximum force on the fracture toughness, the sample deposited at the highest temperature was tested using different maximum forces $P_{\max }$. Figure 15 shows that the crack length is proportional to $P^{2 / 3}$, indicating that the fracture toughness measurement is not influenced by the applied force up to $1 \mathrm{~N}$.

The results of the FEM model are compared to the solution of Anstis et al. [24] and Jang and Pharr [29]. Therefore, $G_{\text {Ic }}$ is varied within the FEM model and the corresponding crack lengths are evaluated. The calculated crack shapes are shown in form of the contact status in the cracking zone in Fig. 16.

Then the obtained crack length is plotted against the fracture toughness and compared to the results of Jang and Pharr [29] and Anstis et al. [24] in Fig. 17.

The solution of the FEM model lies right between the solution obtained by Jang and Pharr [29] and Anstis

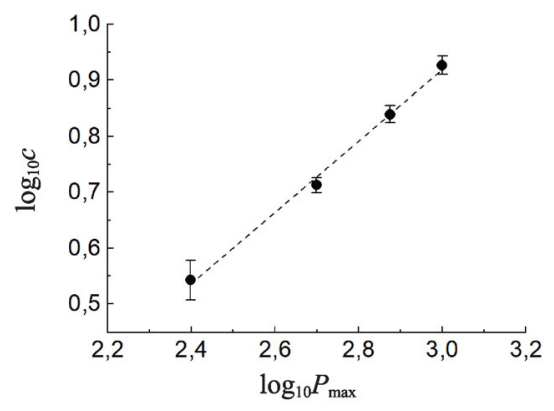

Fig. 15 Log-log plot of the crack length $(c)$ vs. the maximum load $P$.

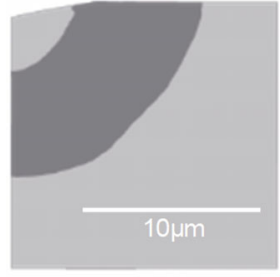

$G_{\mathrm{lc}}=10 \mathrm{~J} / \mathrm{m}^{2}$

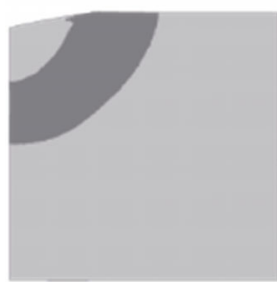

$G_{\mid c}=30 \mathrm{~J} / \mathrm{m}^{2}$

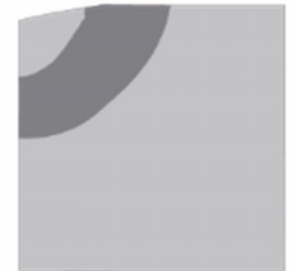

$G_{\mid c}=20 \mathrm{~J} / \mathrm{m}^{2}$

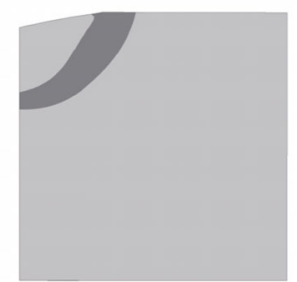

$G_{\mathrm{lc}}=40 \mathrm{~J} / \mathrm{m}^{2}$
Fig. 16 FEM results of the crack shape with parametric critical strain energy release rate, where the light gray zones are still in contact, and the dark shaded gray represents debonded zones.

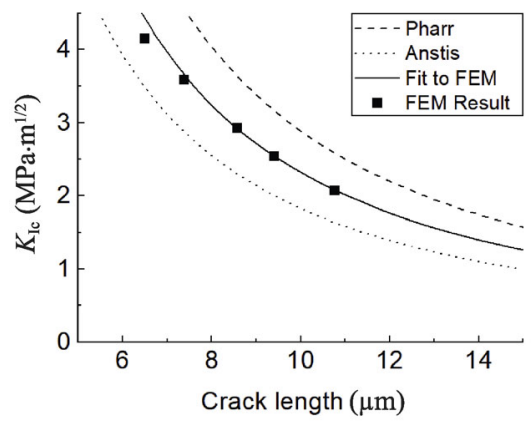

Fig. 17 Fracture toughness as a function of the length of a crack generated by instrumented indentation for different fitting constants (Jang and Pharr 0.025, Anstis et al. 0.016, this study).

et al. [24]. The values obtained by the FEM model were fitted using the same equation as proposed by Anstis et al. A fitting constant of approximately 0.02 gives the best result. This value lies within the tolerances of the constant provided by Anstis et al.

It should be mentioned that, for sufficiently high fracture toughness no well-developed halfpenny crack can be achieved within the simulation. Accordingly, the FEM solution deviates from the fit curve at higher fracture toughness.

Also, the influence of residual stress on the measurement was checked within the FEM model. The numerical results agree very well with the analytical correction from Eq. (10) as can be seen in Fig. 18.

To investigate the influence of the deposition temperature on the fracture toughness, coatings deposited at three different temperatures are used. Figure 19 shows the values of $K_{\text {Ic }}$ for the three different coatings, determined by Eq. (8) and Eq. (10) respectively. For the evaluation the fitting constant used in Fig. 17 was used. The crack length was taken from the SEM measurements. The hardness of the coating was also

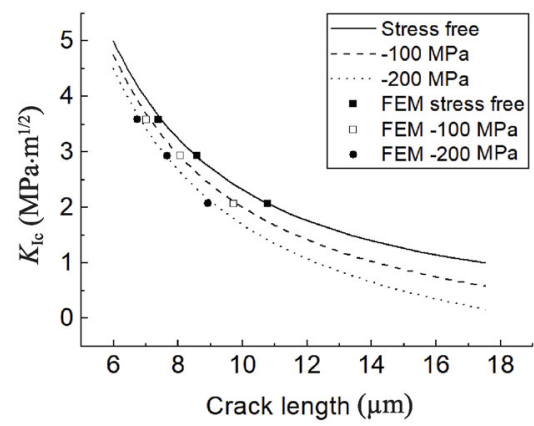

Fig. 18 Influence of residual stress on the fracture toughness determined by instrumented indentation for a sample without residual stress and with compressive residual stress of 100 and $200 \mathrm{MPa}$. 


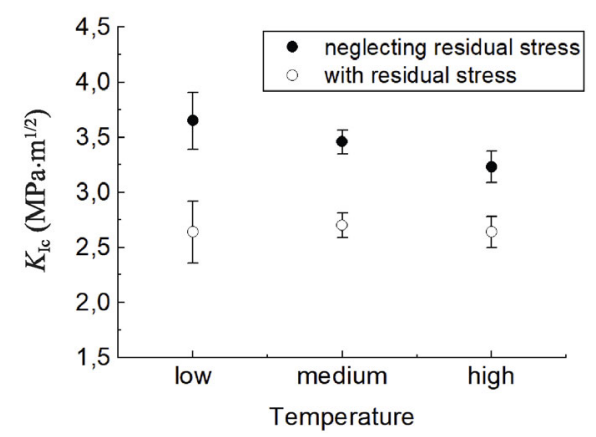

Fig. 19 Fracture toughness of CVD SiC coatings deposited at different temperatures with and without taking residual stress into account.

measured via nanoindentation. The hardness of the SiC coating, varying between 30 and $40 \mathrm{GPa}$, was found to be high compared to the graphite. The aim of this work was to characterize the coating for appropriate material models for FEM simulation. Since hardness does not provide any value in this context, we did not include this in the paper.

Using Eq. (8), the fracture toughness apparently depends on the deposition temperature. If extrinsic residual stresses induced by the differences of the CTE of the coating and the substrate are taken into account (Eq. (10)), this dependence vanishes. The intrinsic residual stress, caused by growth process, is not included in this study and for more insights concerning this topic further experiments are needed. For a more precise analysis of the fracture toughness one would need to use refined stress measurement techniques, or produce coatings detached from the substrate, so residual stress can relax.

Snead et al. [36] give an overview of measurement of the fracture toughness of $\mathrm{SiC}$ from different authors at different $\mathrm{CVD} \mathrm{SiC}$ samples, which range from 2.4 up to $5.1 \mathrm{MPa} \cdot \mathrm{m}^{1 / 2}$ [36]. Compared to these values the fracture toughness seems on the lower end of the reported data with a mean value of $2.7 \mathrm{MPa} \cdot \mathrm{m}^{1 / 2}$ over all measured samples. This could possibly be a result of the used residual stress assumptions, where intrinsic stress effects are neglected. Additionally, the grinding of the surface could have induced residual surface stresses. Therefore, slightly higher values might also be possible.

\section{Discussion}

Nanoindentation measurements normal to the coating surface and in cross section revealed that, the sample has a lower in plane stiffness than out of plane. This might result from the microstructure of the coating, as SEM images show strongly elongated grains in growth direction. This is in good agreement with other investigations of CVD SiC coatings [37,38]. Nevertheless, the mechanical properties can be well described by an isotropic FEM model developed here, which describes the indentation process. The apparent anisotropy of the coating is reflected in the different values of the modulus obtained from the simulations addressing cross sectional and measurements perpendicular to the coating surface.

It could be argued that a transverse isotropic material model of the $\mathrm{SiC}$ coating might be best suited to describe the elastic behavior of the coating. However, it is challenging to determine the material parameters necessary to generate such a model employing nanoindentation. In addition to the 5 independent elastic constants characterizing the transvers isotropic material, information about the anisotropic plastic behavior would be needed. The available experimental data from the nanoindentation experiments would not be sufficient for a unique description of the material properties within such a model. Under these circumstances the application of an isotropic model employing cross-sectional measured values appears preferable, since this allows an easier link to practical application.

In addition to the observed anisotropy, cross-sectional nanoindentation measurements and the measurements normal to the coating surface show that, the deposition temperature has a significant influence on the modulus of the coatings.

The reason for this does not become completely clear. It could be a result of the microstructure of the coating, since the microstructure of coatings deposited at low temperatures deviates significantly from those deposited at medium and high temperature. While the coating deposited at lower temperatures is very finely grained and has a higher modulus, the coatings deposited at higher temperatures show a coarser grain structure coupled with a lower modulus. However, according to Snead et al. [36] no influence of the grain size and the polytype of $\mathrm{SiC}$ on the Young's modulus is expected. Since the higher modulus at lower deposition temperatures can be observed by cross-sectional measurements as well as by measurements normal to the coating surface, the anisotropy and grain orientation can be excluded as a reason for the higher modulus.

Most likely the lower measured modulus is due to 
porosity. While there is no detectable porosity for the coatings deposited at the lowest deposition temperature, porosity is observed for the coatings deposited at higher temperatures. It should be noted, though, that the coating is still very dense. Of course, the higher the porosity in a material, the lower the apparent Young's modulus will be [36]. Due to the low porosity, the quantification of the porosity is a challenging task. Using image processing, no porosity is observed in the low temperature coating, but a porosity of $1 \%-2 \%$ is found in the medium and high deposition temperature coating. The observed porosity and the corresponding lower elastic modulus agrees quantitatively very well with the observed influence of porosity on the elastic modulus of CVD silicon carbide described in literature [36].

Finally, a FEM model was generated to develop a deeper understanding of the fracture process of $\mathrm{SiC}$ during indentation. The FEM model using data generated from our experiments was used to calibrate a fitting constant for the evaluation of the fracture toughness by instrumented indentation. The resulting fracture toughness shows no significant influence of the deposition temperature on the fracture toughness of the material. Typically, one would assume a higher influence of the microstructure on the fracture toughness than on the elastic properties. We assume that the higher impact on the elastic properties originates from the porosity. The indents for fracture toughness investigation were on a scale, where the larger pores might not influence the fracture process itself, therefore leading to no high change in the toughness. We also observed trans granular fracture, which further leads to a lower sensitivity to the grain structure. However, the model reveals that, the determination of fracture toughness via indentation is sensitive to the residual stress in the material, which is often not exactly known. This should be taken into account, when determining fracture toughness of coatings subject to residual stress.

\section{Conclusions}

Silicon carbide coatings were deposited on a graphite substrate by chemical vapor deposition, at different deposition temperatures leading to different microstructure of the coating. Within this study the elastic modulus and the fracture toughness of the coatings were investigated using nanoindentation combined with finite element method-based evaluation procedures. The FEM based evaluation allowed to fully account for the influence of the substrate material on the measurement of the coating properties. Additionally, using the FEM approach for the evaluation of the fracture toughness via indentation allowed us to analyze the fracture toughness without relying on empirical constants. We observed that lower deposition temperatures of the coating led to coatings with higher elastic moduli. For the fracture toughness no significant influence of the deposition temperature was found.

\section{Acknowledgements}

This work was founded by SGL Carbon GmbH. The experimental part of the study was conducted at Department of Experimental Physics II, University of Augsburg. Special thanks to Wolfgang Müller and Michael Schulz for supporting the experimental works.

\section{References}

[1] Chiu CC, Case ED. Elastic modulus determination of coating layers as applied to layered ceramic composites. Mater Sci Eng: A 1991, 132: 39-47.

[2] Watkins TR, Green DJ, Ryba ER. Determination of Young's modulus in chemically vapor-deposited $\mathrm{SiC}$ coatings. J Am Ceram Soc 1993, 76: 1965-1968.

[3] Cheng DJ, Shyy WJ, Kuo DH, et al. Growth characteristics of CVD beta-silicon carbide. J Electrochem Soc 1987, 134: 3145-3149.

[4] Saigal A, Das N. Effect of deposition parameters on the strength of CVD $\beta-S i C$ coatings. Adv Ceram Mater 1988, 3: $580-583$.

[5] Chin J, Gantzel PK, Hudson RG. The structure of chemical vapor deposited silicon carbide. Thin Solid Films 1977, 40: $57-72$.

[6] Gulden TD. Deposition and microstructure of vapor-deposited silicon carbide. J Am Ceram Soc 1968, 51: 424-428.

[7] Oliver WC, Pethica JB. Method for continuous determination of the elastic stiffness of contact betweentwo bodies. United States Patent 4848141. 1989.

[8] Tan L, Allen TR, Hunn JD, et al. EBSD for microstructure and property characterization of the SiC-coating in TRISO fuel particles. J Nucl Mater 2008, 372: 400-404.

[9] International Organisation for Standardization. Metallische Werkstoffe-Instrumentierte Eindringprüfung zur Bestimmung der Härte und anderer Werkstoffparameter- Teil 1: Prüfverfahren (ISO 14577-1:2015); Deutsche Fassung EN ISO 14577-1: 2015 (Foreign Standard). 2015.

[10] Oliver WC, Pharr GM. Measurement of hardness and 
elastic modulus by instrumented indentation: Advances in understanding and refinements to methodology. $J$ Mater Res 2004, 19: 3-20.

[11] Oliver WC, Pharr GM. An improved technique for determining hardness and elastic modulus using load and displacement sensing indentation experiments. J Mater Res 1992, 7: 1564-1583.

[12] Sneddon IN. The relation between load and penetration in the axisymmetric boussinesq problem for a punch of arbitrary profile. Int J Eng Sci 1965, 3: 47-57.

[13] Pharr GM, Oliver WC, Brotzen FR. On the generality of the relationship among contact stiffness, contact area, and elastic modulus during indentation. J Mater Res 1992, 7: 613-617.

[14] Hay J. Webinar: Instrumented Indentation-Session 3: Basic instrumented indentation to measure hardness and Young's modulus. Nanomechanics, Inc, 2016. Available at https:// nanomechanicsinc.webex.com/mw3200/mywebex/default. do? siteurl=nanomechanicsinc.

[15] ANSYS ${ }^{\circledR}$ Mechanical Enterprise, Release 19.0.

[16] Knapp JA, Follstaedt DM, Myers SM, et al. Finite-element modeling of nanoindentation. J Appl Phys 1999, 85: 1460-1474.

[17] Lichinchi M, Lenardi C, Haupt J, et al. Simulation of Berkovich nanoindentation experiments on thin films using finite element method. Thin Solid Films 1998, 312: 240-248.

[18] Bolshakov A, Pharr GM. Influences of pileup on the measurement of mechanical properties by load and depth sensing indentation techniques. J Mater Res 1998, 13: 1049-1058.

[19] Chudoba T, Jennett NM. Higher accuracy analysis of instrumented indentation data obtained with pointed indenters. J Phys D: Appl Phys 2008, 41: 215407.

[20] Yu N, Polycarpou AA, Conry TF. Tip-radius effect in finite element modeling of sub-50 nm shallow nanoindentation. Thin Solid Films 2004, 450: 295-303.

[21] Palmqvist S. A method to determine the toughness of brittle materials, especially hard materials. Jernkontorets Ann 1957, 141: 303-307. (in Swedish)

[22] Lawn BR, Evans AG, Marshall DB. Elastic/plastic indentation damage in ceramics: The median/radial crack system. J Am Ceram Soc 1980, 63: 574-581.

[23] Ćorić D, Ćurković L, Majić Renjo M. Statistical analysis of vickers indentation fracture toughness of Y-TZP ceramics. Trans FAMENA 2017, 41: 1-16.

[24] Anstis GR, Chantikul P, Lawn BR, et al. A critical evaluation of indentation techniques for measuring fracture toughness: I, direct crack measurements. J Am Ceram Soc 1981, 64: 533-538.

[25] Marshall DB, Lawn BR. An indentation technique for measuring stresses in tempered glass surfaces. $J$ Am Ceram Soc 1977, 60: 86-87.
[26] Lawn BR, Fuller ER. Equilibrium penny-like cracks in indentation fracture. J Mater Sci 1975, 10: 2016-2024.

[27] Marshall DB, Lawn BR. Residual stress effects in sharp contact cracking. J Mater Sci 1979, 14: 2001-2012.

[28] Evans AG, Charles EA. Fracture toughness determinations by indentation. J Am Ceram Soc 1976, 59: 371-372.

[29] Jang JI, Pharr GM. Influence of indenter angle on cracking in $\mathrm{Si}$ and Ge during nanoindentation. Acta Mater 2008, 56: 4458-4469.

[30] Quinn GD, Bradt RC. On the vickers indentation fracture toughness test. J Am Ceram Soc 2007, 90: 673-680.

[31] Zhang S, Zhang XM. Toughness evaluation of hard coatings and thin films. Thin Solid Films 2012, 520: 2375-2389.

[32] Hillerborg A, Modéer M, Petersson PE. Analysis of crack formation and crack growth in concrete by means of fracture mechanics and finite elements. Cem Concr Res 1976, 6: 773-781.

[33] Bazant ZP, Ignacio C. Creep and shrinkage of concrete. In: Proceedings of the 5th International RILEM Symposium, 1993.

[34] Weibull W. A statistical distribution function of wide applicability. Journal of Applied Mechanics 1951, 153: 293-297.

[35] Weibull W. The phenomenon of rupture in solids. In: Proceedings of Royal Swedish Institute of Engineering Research 153, 1939.

[36] Snead LL, Nozawa T, Katoh Y, et al. Handbook of SiC properties for fuel performance modeling. $J$ Nucl Mater 2007, 371: 329-377.

[37] Liu Z, Bao YW, Nie GL. Determining anisotropic Young's modulus of ceramic coatings by the relative method. Key Eng Mater 2017, 726: 100-104.

[38] Tan J, Meadows PJ, Zhang D, et al. Young's modulus measurements of $\mathrm{SiC}$ coatings on spherical particles by using nanoindentation. J Nucl Mater 2009, 393: 22-29.

Open Access This article is licensed under a Creative Commons Attribution 4.0 International License, which permits use, sharing, adaptation, distribution and reproduction in any medium or format, as long as you give appropriate credit to the original author(s) and the source, provide a link to the Creative Commons licence, and indicate if changes were made.

The images or other third party material in this article are included in the article's Creative Commons licence, unless indicated otherwise in a credit line to the material. If material is not included in the article's Creative Commons licence and your intended use is not permitted by statutory regulation or exceeds the permitted use, you will need to obtain permission directly from the copyright holder.

To view a copy of this licence, visit http://creativecommons.org/licenses/by/4.0/. 